\title{
Differences in the Mechanisms by Which Yang-Invigorating and Qi-Invigorating Chinese Tonifying Herbs Stimulate Mitochondrial ATP Generation Capacity
}

\author{
Pou Kuan Leong, Hoi Yan Leung, Wing Man Chan, Kam Ming Ko* \\ Division of Life Science, The Hong Kong University of Science and Technology, Hong Kong, China \\ Email: ^bcrko@ust.hk
}

How to cite this paper: Leong, P.K., Leung, H.Y., Chan, W.M. and Ko, K.M. (2018) Differences in the Mechanisms by Which Yang-Invigorating and Qi-Invigorating Chinese Tonifying Herbs Stimulate Mitochondrial ATP Generation Capacity. Chinese Medicine, 9, 63-74.

https://doi.org/10.4236/cm.2018.92005

Received: May 14, 2018

Accepted: June 10, 2018

Published: June 13, 2018

Copyright $\odot 2018$ by authors and Scientific Research Publishing Inc. This work is licensed under the Creative Commons Attribution International License (CC BY 4.0).

http://creativecommons.org/licenses/by/4.0/

\section{(c) (i) Open Access}

\begin{abstract}
According to Chinese medicine theory, Yang/Qi plays a pivotal role in driving physiological functions in the body, these being highly dependent on mitochondrial ATP production. Consistent with this, Yang/Qi-invigorating Chinese tonifying herbs have been found to stimulate mitochondrial ATP generation capacity (ATP-GC) in $\mathrm{H} 9 \mathrm{c} 2$ cardiomyocytes. In the present study, we have demonstrated that Yang-invigorating Chinese tonifying herbs (namely, Eucommiae Cortex, Cibotii Rhizoma, Dipsaci Radix, Cynomorii Herba, Cistanches Herba, Cuscutae Semen, EpimediiHerba and Morindae Radix) and Qi-invigorating Chinese tonifying herbs (namely, Ginseng Radix, Pseudostellariae Radix, Quinquefolii Radix, Codonopsis Radix, Astragali Radix, Atractylodis Rhizoma, Juiubae Fructus, Fici Simplicissimae Radix and Dioscoreae Oppositae Radix) act by different mechanisms to stimulate mitochondrial ATP-GC. While Yang-invigorating herbs fluidize mitochondrial membranes and thus stimulate ATP-GC, Qi-invigorating herbs can enhance cellular glutathione status and increase ATP-GC. The different mechanisms by which Yang-invigorating herbs and Qi-invigorating herbs stimulate mitochondrial ATP-GC may serve as the basis for establishing biomarkers for Yang/Qi-invigorating herbs and herbal health products in general.
\end{abstract}

\section{Keywords}

Yang-Invigoration, Qi-Invigoration, ATP Generation Capacity, Glutathione

\section{Introduction}

The Yin-Yang Theory, which is a fundamental conceptual framework of Chinese 
medicine, views life activities as a result of an interaction between two complementary but opposing forces, namely, Yin and Yang. The dynamic equilibrium between Yin and Yang enables the attainment of a balance of physiological functions in the body and hence a state of optimal health [1]. According to Chinese medicine theory, functional activities in the body are driven by Yang/Qi, while Yin/Blood forms the material basis for maintaining vital functions such as those of cardiovascular system and immune system [2]. With any imbalance of Yin and Yang, the generation of Qi and Blood from their interaction in the body, can lead to the development of various "deficiency" states relating to Yang, Qi, Yin and Blood components. A "deficiency" state, which is referred to a sub-healthy status in Western medicine, can result from a genetic predisposition, acquiring environmental and lifestyle factors, as well as aging. To avoid the worsening of an unhealthy or "deficient" body condition, it is important to remedy this by restoring Yang, Qi, Yin and Blood components to normal levels. In doing so, Chinese tonifying herbs, which are divided into four functional groups (namely Yang-invigorating, Qi-invigorating, Yin-nourishing and Blood-enriching) [3], have been used for safeguarding and promoting health since ancient times.

As mentioned earlier, Yang and Qi are manifestations of various body functions, all of which are dependent on cellular activities. In this regard, ATP, which is generated by mitochondria (the "power house" of the cell), energizes a wide range of cellular activities. To sustain the capacity to generate ATP, mitochondria are equipped with a glutathione-dependent antioxidant system to protect mitochondrial respiratory complexes against reactive oxygen species (ROS) arising from mitochondrial electron transport processes [4] [5]. In this regard, the Yang/Qi-invigorating action of Chinese tonifying herbs may be related to the stimulation of mitochondrial ATP generation and/or the induction of a glutathione antioxidant response that can preserve mitochondrial ATP generation capacity. Our laboratory has previously demonstrated that Yang-invigorating herbs can increase ATP-generation capacity in cultured H9c2 cardiomyocytes in vitro as well as rat heart mitochondria ex vivo [6] [7], presumably by fluidizing the mitochondrial inner membrane [8]. However, whether or not Qi-invigorating herbs can stimulate mitochondrial ATP generation and the underlying biochemical mechanism(s) involved have yet to be investigated. In the present study, we studied the effects of short-term $(4 \mathrm{~h})$ and long-term exposure (24 h) to methanolic extracts of Yang-invigorating and Qi-invigorating herbs on mitochondrial ATP generation capacity (ATP-GC) and cellular reduced glutathione (GSH) levels (which are indicative of a glutathione antioxidant response) in cultured $\mathrm{H} 9 \mathrm{c} 2$ cardiomyocytes. To investigate the role of membrane fluidization in the stimulation of mitochondrial ATP generation, as in the case for Yang-invigorating herbs, cholesterol was used as a pharmacological tool for stabilizing membrane lipids. To investigate the possible cause and consequence relationship between the stimulation of ATP generation capacity and the induction a glutathione antioxidant response, as in the case for Qi-invigorating herbs, specific inhibitors of glutathione synthesis and regeneration were used. 


\section{Materials and Methods}

\subsection{Chemicals and Reagents}

Dulbecco's Modified Eagle's medium (DMEM) and fetal bovine serum (FBS) were purchased from Gibco BRL Life Technologies (Grand Island, NY, USA). Adenosine diphosphate (ADP), adenosine triphosphate (ATP), sodium pyruvate and sodium malate, the reduced form of glutathione (GSH) and the oxidized form of glutathione (GSSG) were bought from Sigma Chemical Co. (St. Louis, MO, USA). All other chemicals were of analytical grade.

\subsection{Preparation of Herbal Extracts}

Yang-invigorating herbs and Qi-invigorating herbs (see Table 1) were purchased from a local herbal dealer (Lee HoongKee Limited, HKSAR, China). The herbs were authenticated by the supplier and voucher specimens were deposited in the Division of Life Science, Hong Kong University of Science and Technology. Each herb was extracted with methanol under reflux for $2 \mathrm{~h}$, as previously described [9], to obtain amethanolic extract. Extracts were dried by evaporation under reduced pressure and the dried extracts, which were obtained at various yields with reference to the crude herb (see Table 1), were stored at $20^{\circ} \mathrm{C}$ prior to use.

Table 1. Yang-invigorating and Qi-invigorating Chinese tonifying herbs.

\begin{tabular}{|c|c|c|c|}
\hline & Pharmaceutical name & Abbreviation & $\%$ yield \\
\hline \multicolumn{4}{|l|}{ Yang-invigorating herbs } \\
\hline & Eucommiae Cortex & $\mathrm{EC}$ & 12.1 \\
\hline & Cibotii Rhizoma & CR & 23.0 \\
\hline & Dipsaci Radix & DR & 35.0 \\
\hline & Cynomorii Herba & $\mathrm{CYH}$ & 15.5 \\
\hline & Cistanches Herba & $\mathrm{CIH}$ & 26.0 \\
\hline & Cuscutae Semen & CS & 4.24 \\
\hline & Epimedii Herba & $\mathrm{EH}$ & 9.00 \\
\hline & Morindae Radix & MR & 29.9 \\
\hline \multicolumn{4}{|l|}{ Qi-invigorating herbs } \\
\hline & Ginseng Radix & GR & 13.0 \\
\hline & Pseudostellariae Radix & $\mathrm{PR}$ & 47.3 \\
\hline & Quinquefolii Radix & QR & 12.0 \\
\hline & Codonopsis Radix & $\mathrm{CR}$ & 37.2 \\
\hline & Astragali Radix & ASR & 27.2 \\
\hline & Atractylodis Rhizoma & ATR & 20.5 \\
\hline & Juiubae Fructus & $\mathrm{JF}$ & 52.7 \\
\hline & Fici Simplicissimae Radix & FR & 3.60 \\
\hline & Dioscoreae Oppositae Radix & DOR & 53.1 \\
\hline
\end{tabular}




\subsection{Cell Culture}

H9c2 cells, which area subclone of rat heart myoblast cells, were purchased from the American Tissue Culture Centre (ATCC). Cells were cultured as mono-layers in DMEM, supplemented with $10 \%(\mathrm{v} / \mathrm{v}) \mathrm{FBS}, 100 \mathrm{IU} / \mathrm{mL}$ of penicillin, 100 $\mu \mathrm{g} / \mathrm{mL}$ of streptomycin and $1.5 \mathrm{~g} / \mathrm{L} \mathrm{NaHCO}_{3}$. All cells were grown under an atmosphere of $5 \%(\mathrm{v} / \mathrm{v}) \mathrm{CO}_{2}$ in air at $37^{\circ} \mathrm{C}$.

\subsection{Herbal Extract Incubation}

H9c2 cardiomyocytes were seeded at a density of $1 \times 10^{5}$ cells/well into 12 -well microtiter plates or at $5 \times 10^{4}$ cells/well into 24-well microtiter plates. Following attachment, cells were incubated with Yang or Qi-invigorating herbal extracts (at a concentration of $100 \mu \mathrm{g} / \mathrm{mL}$ ) for $4 \mathrm{~h}$ or $24 \mathrm{~h}$ at $37^{\circ} \mathrm{C}$. The herbal extract-containing medium was prepared by adding the sample solution in dimethyl Sulfoxide (DMSO) to the culture medium, with the final concentration of DMSO being less than $0.2 \%(\mathrm{v} / \mathrm{v})$. The control group received vehicle [DMSO $(0.2 \%, \mathrm{v} / \mathrm{v}$, final concentration $)]$ only.

\subsection{Incubations with Inhibitors}

To determine the role of membrane fluidity in the effect of the Yang-invigorating herbal extract on mitochondrial ATP-GC, cholesterol [ $3 \mu \mathrm{M}$, dissolved in ethanol, the final concentration of solvent in the medium being $0.2 \%(\mathrm{v} / \mathrm{v})]$, which can decrease mitochondrial inner membrane fluidity [10], was co-incubated with the Yang-invigorating herbal extract $(100 \mu \mathrm{g} / \mathrm{mL})$ for $4 \mathrm{~h}$ in $\mathrm{H} 9 \mathrm{c} 2$ cells.

To determine the role of a glutathione-dependent antioxidant response in the stimulation of mitochondrial ATP-GC by Qi-invigorating herbal extracts, H9c2 cells were co-incubated with L-buthionine-(S, R)-sulfoximine (BSO, which blocks glutathione synthesis by inhibiting $\gamma$-glutamylcysteine synthetase, at 12.5 $\mu \mathrm{M})$ and Qi-invigorating herbal extracts $(100 \mu \mathrm{g} / \mathrm{mL})$ for $24 \mathrm{~h}$, and $\mathrm{N}, \mathrm{N}^{\prime}$-bis (2-chloroethyl)-N-nitrosourea [BCNU, a potent inhibitor of glutathione reductase, at $25 \mu \mathrm{M}$ in $0.2 \%$ ethanol in the culture medium (v/v)] was added $(2 \mu \mathrm{L})$ in the incubation mixture $1 \mathrm{~h}$ prior to the end of the $24-\mathrm{h}$ incubation.

\subsection{ATP Generation Capacity in Situ}

Following the incubation with the herbal extract, the mitochondrial ATP-GC assay was performed as previously described [6] [7] [9]. In brief, the removal of Yang- or Qi-invigorating herbal extract-containing medium was followed by cell membrane permeabilization with digitonin $(50 \mu \mathrm{g} / \mathrm{mL})$ in an incubation buffer (120 mM KCl, $5 \mathrm{mM} \mathrm{KH} \mathrm{PO}_{4}, 2 \mathrm{mM}$ EGTA, $10 \mathrm{mM}$ HEPES, $0.1 \mathrm{mM} \mathrm{MgCl}$, $0.5 \%$ BSA, pH 7.4). Pyruvate ( $15 \mathrm{mM})$, malate $(5 \mathrm{mM})$ and ADP $(10 \mu \mathrm{M})$ were added to initiate mitochondrial ATP generation at $37^{\circ} \mathrm{C}$, which was monitored at increasing time intervals ranging from 0 to $15 \mathrm{~min}$. The reaction was terminated by the addition of $60 \mu \mathrm{L}$ of perchloric acid $(30 \%, w / v)$, and the reaction mixtures were then centrifuged at $540 \times \mathrm{g}$ for $10 \mathrm{~min}$ at $4^{\circ} \mathrm{C}$, and the superna- 
tants were measured for ATP content by the luciferase assay (ATPlite, PerkinElmer Inc., MA, USA). Mitochondrial ATP-GC in the non-herbal pre-incubated control was determined by computing the area under the curve (AUC1) of the graph plotting ATP generated (nmol/mg protein) versus time (0, 7.5 and 15 $\mathrm{min}$ ) and expressed in arbitrary units. For herbal extract-pre-incubated samples, AUC1 values at increasing incubation times were normalized to a respective mean control value from non-herbal-pre-incubated cells and expressed as a percent of control. The area under the curve (AUC2) of a graph plotting percent control values versus incubation time $(0,7.5$ and $15 \mathrm{~min})$ was calculated and expressed in arbitrary units. Data for herbal extract-pre-incubated groups were expressed as a percent of control. The two-step data processing aimed at minimizing inter-animal and inter-assay variabilities under the present experimental conditions [6] [7] [9].

\subsection{GSH Level}

After the incubation with herbal extract, the cellular level of GSH was measured using the method of Griffith [11]. In brief, the total glutathione content of cells cultured in a 12-well microtiter plate was preserved by the addition of $100 \mu \mathrm{L} 3 \%$ 5 -sulfosalicylic acid (SSA, w/v), i.e. the total glutathione sample. To measure the GSSG level, $50 \mu \mathrm{L}$ of total glutathione sample were mixed with $5 \mu \mathrm{L}$ of $20 \%(\mathrm{w} / \mathrm{v})$ 2 -vinylpyridine and $5 \mu \mathrm{L}$ of $60 \%(\mathrm{w} / \mathrm{v})$ triethanolamine in microcentrifuge tubes. Each tube was allowed to stand at room temperature for $30 \mathrm{~min}$ for the derivatization of GSH. An aliquot of $180 \mu \mathrm{L}$ reaction cocktail containing $0.63 \mathrm{mM}$ 5,5-dithio-bis-2-nitrobenzoic acid, $0.053 \mathrm{mM}$ nicotinamide adenine dinucleotide phosphate (reduced form, NADPH) and $0.525 \mathrm{U} / \mathrm{mL}$ glutathione reductase in phosphate buffer ( $0.1 \mathrm{M}$ with $5 \mathrm{mM}$ ethylene diaminetetraacetic acid, $\mathrm{pH} 7.5$ ) was mixed with $30 \mu \mathrm{L}$ of the total glutathione sample or the GSSG sample in a 96-well microtiter plate. Absorbance changes at $405 \mathrm{~nm}$ of each reaction mixture were monitored spectrophotometrically by Victor V3 Multi-label Counter (Perkin Elmer, Turku, Finland). The concentrations of total glutathione and GSSG were estimated from calibration curves using GSH or GSSG as standards and expressed as nmol/mg protein. Cellular GSH levels were estimated by subtracting twice the amount of GSSG from that of total glutathione.

\subsection{Protein Assay}

Protein concentration was determined with a Bio-Rad protein assay kit (Bio-Rad, Hercules, CA), using bovine serum albumin as standard.

\subsection{Statistical Analysis}

All data were expressed as mean \pm standard error of the mean (SEM), unless otherwise specified. Data were analyzed by Student's t-test or one-way analysis of variance (one-way Anova), as specified. For one-way Anova, inter-group differences were detected by Tukey, with $\mathrm{p}<0.05$. The correlation between GSH 
levels and mitochondrial ATP-GC values after $24 \mathrm{~h}$ incubation with Qi-invigorating herbal extracts was analyzed by computing the Pearson's correlation coefficient using the two-tailed significance test.

\section{Results}

\subsection{Effect of Yang-Invigorating Herbal Extracts on Mitochondrial ATP-GC and Cellular GSH Levels in H9c2 Cells}

A 4-h incubation with all tested Yang-invigorating herbal extracts consistently increased mitochondrial ATP-GC in H9c2 cells (ranging from 13\% - 41\%, Table 2). However, prolonged incubation (i.e. $24 \mathrm{~h}$ ) with Yang-invigorating herbal extracts produced different effects on mitochondrial ATP-GC, as indicated by decreased extents of mitochondrial ATP-GC stimulation (CYH, CIH and CS), no mitochondrial ATP-GC stimulation (CR, DR, EH and MR) or even a suppression of mitochondrial ATP-GC stimulation (EC, a 30\% decrease when compared with the non-herbal-pre-incubated controls). The ability of Yang-invigorating herbal extracts ( $4 \mathrm{~h}$ pre-incubation) to increase mitochondrial ATP-GC was completely abrogated by co-incubation with cholesterol (Figure 1). In the case of DR, co-incubation with cholesterol caused a decrease in mitochondrial

Table 2. Effects of Yang-invigorating and Qi-invigorating herbal extracts on mitochondrial ATP-GC in H9c2 cells.

\begin{tabular}{|c|c|c|}
\hline & 4-h incubation & 24-h incubation \\
\hline \multicolumn{3}{|c|}{ Yang-invigorating herbal extracts } \\
\hline EC & $120 \pm 6.46^{*}$ & $69.1 \pm 5.91^{\star}$ \\
\hline CR & $113 \pm 4.32^{*}$ & $104 \pm 2.67$ \\
\hline DR & $124 \pm 8.79^{*}$ & $102 \pm 7.17$ \\
\hline $\mathrm{CYH}$ & $131 \pm 6.44^{*}$ & $105 \pm 3.08^{*}$ \\
\hline $\mathrm{CIH}$ & $141 \pm 10.8^{*}$ & $116 \pm 3.06^{*}$ \\
\hline CS & $121 \pm 3.98^{*}$ & $113 \pm 1.82^{*}$ \\
\hline $\mathrm{EH}$ & $117 \pm 9.77^{\star}$ & $93.8 \pm 6.29$ \\
\hline MR & $117 \pm 3.89^{*}$ & $100 \pm 1.52$ \\
\hline \multicolumn{3}{|c|}{ Qi-invigorating herbal extracts } \\
\hline GR & $109 \pm 4.15^{*}$ & $121 \pm 3.53^{*}$ \\
\hline PR & $122 \pm 7.28^{*}$ & $117 \pm 1.65^{*}$ \\
\hline QR & $93.1 \pm 1.93^{*}$ & $140 \pm 4.71^{\star}$ \\
\hline CR & $94.5 \pm 5.93$ & $132 \pm 3.34^{*}$ \\
\hline ASR & $97.9 \pm 5.72$ & $122 \pm 10.3^{*}$ \\
\hline ATR & $86.3 \pm 5.42^{*}$ & $133 \pm 8.48^{*}$ \\
\hline JF & $115 \pm 5.42^{*}$ & $116 \pm 8.36^{*}$ \\
\hline FR & $97.1 \pm 4.13$ & $132 \pm 3.51^{*}$ \\
\hline DOR & $98.3 \pm 3.96$ & $130 \pm 4.92$ \\
\hline
\end{tabular}

Values given are expressed as \% Control (mean $\pm \mathrm{SD}) .{ }^{*}$ Significantly different from the respective control by Student's $t$ test $(\mathrm{p}<0.05)$. The control value of AUC2 of Yang-invigorating herbal extracts $=750 \pm 35.1$. The control value of AUC2 of Qi-invigorating herbal extracts $=750 \pm 45.0$. 


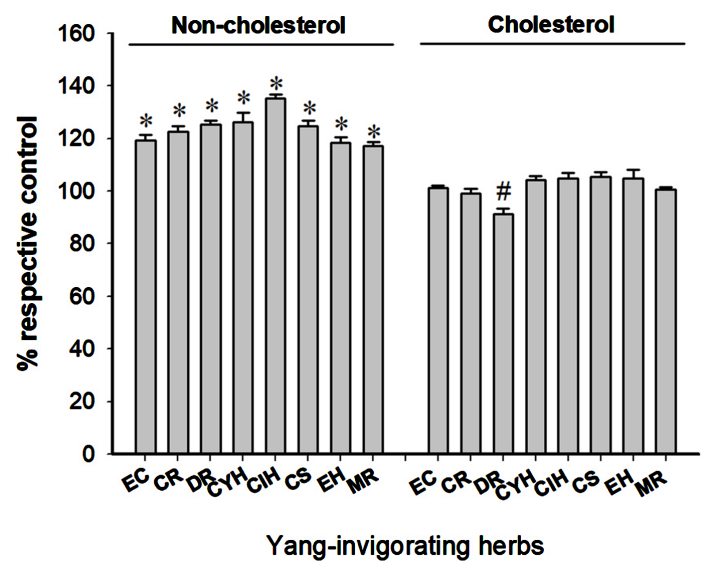

Figure 1. Effect of cholesterol on the stimulation of mitochondrial ATP-GC by Yang-invigorating herbal extracts. H9c2 cardiomyocytes were co-incubated with Yang-invigorating herbal extracts and cholesterol for 4 hours, as described in Materials and Methods. After the 4-h incubation, in situ ATP-GC was measured as described in Materials and Methods. Values given are expressed as \% control (mean $\pm \mathrm{SD}) .{ }^{*}$ Significantly different from the respective control by one-way Anova, Tukey $(\mathrm{p}<0.05)$.

ATP-GC in H9c2 cells, when compared with cholesterol-pre-incubated controls.

None of the tested Yang-invigorating herbal extracts increased cellular GSH levels in H9c2 cells after a 4-h incubation. Following a 24-h incubation with the herbal extracts (EC, CR, CIH, CS and MR), cellular GSH levels were increased, with the degree of stimulation being $14 \%-110 \%$, when compared with the non-herbal-pre-incubated controls (Table 3). No significant correlation between the degree of mitochondrial ATP-GC stimulation (4-h incubation) and the increase in cellular GSH levels (24-h incubation) by the Yang-invigorating herbal extracts was detected (Table 4).

\subsection{Effects of Qi-Invigorating Herbal Extracts on Mitochondrial ATP-GC and Cellular GSH Levels in H9c2 Cells}

Incubation with Qi-invigorating herbal extracts for $4 \mathrm{~h}$ did not produce identical effects on mitochondrial ATP-GC in H9c2 cells (Table 2). While some herbal extracts (GR, PR and JF) significantly increased mitochondrial ATP-GC (9\% - 22\%), QR and ATR significantly decreased mitochondrial ATP-GC (7\% - 14\%). The other extracts (CR, ASR, FR and DOR) did not produce any detectable change in mitochondrial ATP-GC. However, when H9c2 cells were incubated with Qi-invigorating herbal extracts for a longer time-course (i.e. $24 \mathrm{~h}$ ), all tested Qi-invigorating herbal extracts increased mitochondrial ATP-GC, with the extent of stimulation being $16 \%-40 \%$.

Some Qi-invigorating herbal extracts ( $\mathrm{QR}, \mathrm{ASR}$ and $\mathrm{FR})$ were found to enhance cellular GSH levels following a 4-h incubation (12\% - 17\%, Table 3). A longer incubation (i.e. $24 \mathrm{~h}$ ) with Qi-invigorating herbal extracts consistently elicited a glutathione antioxidant response, as indicated by increases in cellular GSH levels (14\% to $46 \%$ ), when compared with the non-herbal-pre-incubated 
Table 3. Effects of Yang-invigorating and Qi-invigorating herbal extracts on cellular GSH levels in $\mathrm{H} 9 \mathrm{c} 2$ cells.

\begin{tabular}{|c|c|c|}
\hline & 4-h incubation & 24-h incubation \\
\hline \multicolumn{3}{|c|}{ Yang-invigorating herbal extracts } \\
\hline EC & $105 \pm 5.37$ & $210 \pm 9.85^{*}$ \\
\hline $\mathrm{CR}$ & $94.4 \pm 5.51$ & $118 \pm 2.41^{*}$ \\
\hline DR & $100 \pm 4.86$ & $104 \pm 4.51$ \\
\hline $\mathrm{CYH}$ & $101 \pm 4.69$ & $105 \pm 6.04$ \\
\hline $\mathrm{CIH}$ & $95.6 \pm 3.06$ & $120 \pm 6.92^{*}$ \\
\hline CS & $101 \pm 3.93$ & $126 \pm 7.86^{*}$ \\
\hline $\mathrm{EH}$ & $99.9 \pm 8.48$ & $102 \pm 4.46$ \\
\hline MR & $104 \pm 1.68$ & $114 \pm 2.55^{\star}$ \\
\hline \multicolumn{3}{|c|}{ Qi-invigorating herbal extracts } \\
\hline GR & $93.9 \pm 2.92$ & $121 \pm 0.61^{*}$ \\
\hline $\mathrm{PR}$ & $106 \pm 4.53$ & $118 \pm 1.67^{*}$ \\
\hline QR & $112 \pm 3.31^{*}$ & $143 \pm 4.04^{*}$ \\
\hline $\mathrm{CR}$ & $98.1 \pm 4.51$ & $129 \pm 4.63^{*}$ \\
\hline ASR & $114 \pm 5.17^{*}$ & $114 \pm 2.29^{*}$ \\
\hline ATR & $106 \pm 3.42$ & $179 \pm 5.13^{*}$ \\
\hline $\mathrm{JF}$ & $103 \pm 4.99$ & $115 \pm 6.81^{*}$ \\
\hline FR & $117 \pm 4.86^{*}$ & $146 \pm 12.7^{*}$ \\
\hline DOR & $106 \pm 8.28$ & $113 \pm 5.74^{*}$ \\
\hline
\end{tabular}

Values given are expressed as \% Control (mean $\pm \mathrm{SD}) .{ }^{*}$ Significantly different from the respective control by Student's test $(\mathrm{p}<0.05)$. The control value of Yang-invigorating herbal extracts $=33.9 \pm 4.76(\mathrm{nmol} / \mathrm{mg}$ protein). The control value of Qi-invigorating herbal extracts $=30.3 \pm 2.47$ ( $\mathrm{nmol} / \mathrm{mg}$ protein).

Table 4. Correlation analyses between Yang-invigorating herbal extract-induced mitochondrial ATP-GC stimulation and cellular GSH increase in $\mathrm{H} 9 \mathrm{c} 2$ cells.

\begin{tabular}{cccc}
\hline & Pearson's Correlation & R squared & p value \\
\hline Yang-invigorating herbal extracts & ATP-GC (4 h) vs cellular GSH (24 h) & 0.0749 & 0.476 \\
Qi-invigorating herbal extracts & ATP-GC (24 h) vs cellular GSH (24 h) & 0.403 & 0.044 \\
\hline
\end{tabular}

controls (Table 3). A moderate but significant correlation between the degree of mitochondrial ATP-GC stimulation and the increase in cellular GSH levels by Qi-invigorating herbal extracts after a 24-h incubation was observed (Table 4). To determine whether the glutathione antioxidant response is causally related to the stimulation of mitochondrial ATP-GC, the effect of Qi-invigorating herbal extracts in $\mathrm{BSO} / \mathrm{BCNU}$-co-incubated $\mathrm{H} 9 \mathrm{c} 2$ cells was examined. Co-incubation with $\mathrm{BSO}$ and $\mathrm{BCNU}$ abrogated both the glutathione antioxidant response (Figure 2(a)) and the stimulation of mitochondrial ATP-GC (Figure 2(b)) by Qi-invigorating herbal extracts following incubation for $24 \mathrm{~h}$. 


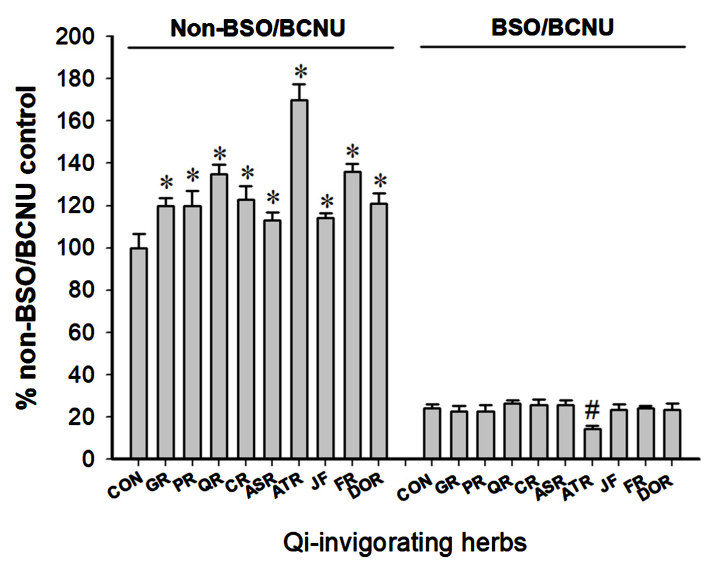

(a)

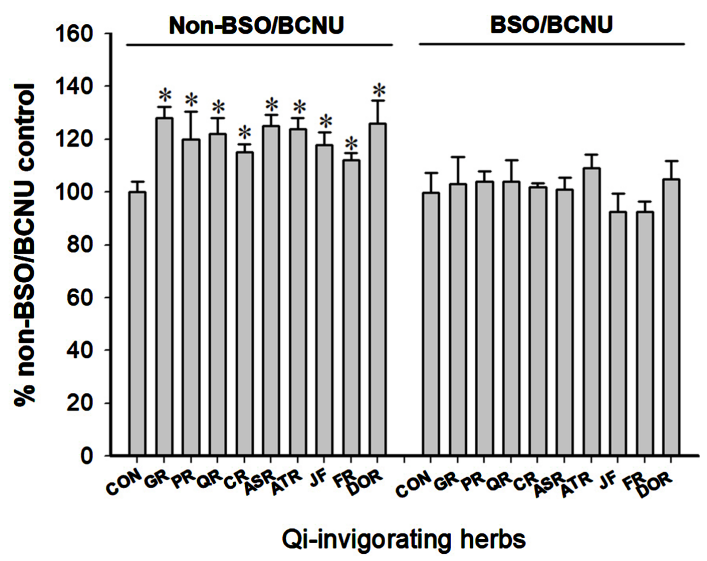

(b)

Figure 2. Effects of cellular GSH suppression on mitochondrial ATP-GC stimulation induced by Qi-invigorating herbal extracts. H9c2 cardiomyocytes were incubated with Qi-invigorating herbal extracts, in the presence of BSO and BCNU, as described in Materials and Methods. After a24-h incubation, cellular GSH levels (a) and in situ ATP-GC (b) were measured as described in Materials and Methods. Values given are expressed as $\%$ control (mean $\pm \mathrm{SD})$. Cellular GSH Control value $\pm \mathrm{SD}(\mathrm{nmol} / \mathrm{mg}$ protein $)=33.6 \pm$ 2.25. ATP-GC Control value \pm SD $($ AUC2 $)=750 \pm 30.5 .{ }^{\star}$ Significantly different from the respective controls by one-way Anova, Tukey $(\mathrm{p}<0.05)$. " Significantly different from the respective BSO/BCNU control by one-way Anova, Tukey $(\mathrm{p}<0.05)$.

\section{Discussion}

According to Chinese medicine theory, Yang/Qi balance plays a pivotal role in modulating physiological functions in the body. The gradual depletion of Yang/Qi is associated with aging and the predisposition to age-related diseases in humans [12]. In this regard, Yang/Qi deficiency can be corrected by using Yang/Qi-invigorating Chinese tonifying herbs for restoring optimal body health. Recent studies in our laboratory have demonstrated the pharmacological basis of Yang-invigoration, wherein Yang-invigorating herbal extracts invariably increased mitochondrial ATP-GC both ex vivo in mouse hearts and in vitro in H9c2 cells [6] [7]. Results obtained from the present study have shown that Qi-invigorating herbal extracts can increase mitochondrial ATP-GC in H9c2 
cells after a prolonged $(24 \mathrm{~h})$ rather than a shorter $(4 \mathrm{~h})$ incubation, as the case for Yang-invigorating herbal extracts. This observation points to the possibility that Yang-invigorating and Qi-invigorating herbs stimulate mitochondrial ATP-GC via different mechanisms. In this connection, an earlier study in our laboratory has demonstrated the involvement of fluidization of the mitochondrial inner membrane in the stimulation of mitochondrial ATP-GC by $\beta$-sitosterol, an active component of Cistanches Herba (a Yang-invigorating tonifying herb), in cultured $\mathrm{C} 2 \mathrm{C} 12$ myocytes [8]. This is consistent with the results obtained from the present study showing that the Yang-invigorating herbal extract-induced stimulation of ATP-GC is completely abrogated by incubation with cholesterol (a biomembrane stabilizer). Presumably, active components of Yang-invigorating herbal extracts act to fluidize the mitochondrial inner membrane and thus activate enzymes involved in electron transport [8], with a resultant increase in mitochondrial ATP-GC in H9c2 calls under the present experimental conditions.

On the other hand, while Qi-invigorating herbal extracts failed to stimulate mitochondrial ATP-GC following a 4-h incubation, they invariably stimulated ATP-GC after incubation for $24 \mathrm{~h}$ in H9c2 cells. The stimulation of mitochondrial ATP-GC was accompanied by an increase in cellular GSH levels. Correlation analysis showed that the degree of mitochondrial ATP-GC stimulation correlated significantly with the extent of cellular GSH increase in Qi-invigorating herbal extract-pre-incubated $\mathrm{H} 9 \mathrm{c} 2$ cells. The finding that $\mathrm{BSO} / \mathrm{BCNU}$ co-incubation abolishes the stimulatory effect of Qi-invigorating herbal extracts on mitochondrial ATP-GC further confirms the proposal that the increase in mitochondrial ATP-GC is an effect secondary to the enhancement of cellular GSH status. This is consistent with earlier findings indicating that glutathione antioxidant status plays a critical role in preserving mitochondrial structural and functional integrity [13] [14]. The pivotal role of enhancement in glutathione redox status in stimulating mitochondrial ATP-GC is further supported by the observation that the incubation with cholesterol $4 \mathrm{~h}$ prior to the end of 24-h incubation with Qi-invigorating herbal extracts did not suppress the increase in mitochondrial ATP-GC in H9c2 cells (data not shown). Recent studies of antioxidant phytochemicals have shown that various phytochemicals (such as curcumin, catechin, resveratrol and schisandrin B) can serve as direct free radical scavengers and/or induce a nuclear factor (erythroid-derived 2)-like 2 (Nrf2)-driven expression of antioxidant proteins/enzymes [15], [16]. With respect to the mechanism underlying the enhancement of cellular GSH levels produced by Qi-invigorating herbs, these may possess free radical scavenging activity with the subsequent preservation of cellular GSH levels and/or they may increase the expression of glutathione-dependent proteins/enzymes.

In conclusion, our findings indicate that Yang-invigorating and Qi-invigorating herbs can both stimulate mitochondrial ATP-GC in H9c2 cells but by different mechanisms. While Yang-invigorating herbs act primarily by fluidizing the inner mitochondrial membrane, Qi-invigorating herbs exert their action indirectly 
by increasing cellular GSH levels. Future studies will focus on the mechanism underlying the capacity of Qi-invigorating herbs to enhance cellular GSH levels. The measurement of mitochondrial ATP-GC and cellular GSH levels in H9c2 cells under the experimental conditions described here could serve as convenient biomarkers for the quality assurance of Yang-invigorating and Qi-invigorating Chinese tonifying herbs and herbal health products in general.

\section{References}

[1] Yin, H. and Shuai, X. (1992) Fundamentals of Traditional Chinese Medicine. Foreign Languages Press, Beijing.

[2] Liu, Z. and Liu, L. (2009) Essentials of Chinese Medicine. Springer, London.

[3] Geng, J., Huang, W., Ren, T. and Ma, X. (1991) Practical Traditional Chinese Medicine and Pharmacology. New World Press, Beijing.

[4] Kolossov, V.L., Beaudoin, J.N., Ponnuraj, N., DiLiberto, S.J., Hanafin, W.P., Kenis P.J., et al. (2015) Thiol-Based Antioxidants Elicit Mitochondrial Oxidation via Respiratory Complex III. American Journal of Physiology-Cell Physiology, 309, C81-C91. https://doi.org/10.1152/ajpcell.00006.2015

[5] Dröse, S., Brandt, U. and Wittig, I. (2014) Mitochondrial Respiratory Chain Complexes as Sources and Targets of Thiol-Based Redox-Regulation. Biochimica et Biophysica Acta (BBA) - Proteins and Proteomics, 1844, 1344-1354. https://doi.org/10.1016/j.bbapap.2014.02.006

[6] Ko, K.M., Leon, T.Y., Mak, D.H., Chiu, P.Y., Du, Y. and Poon, M.K. (2006) A Characteristic Pharmacological Action of "Yang-Invigorating" Chinese Tonifying Herbs: Enhancement of Myocardial ATP-Generation Capacity. Phytomedicine, 13, 636-642. https://doi.org/10.1016/j.phymed.2006.02.007

[7] Ko, K.M. and Leung, H.Y. (2007) Enhancement of ATP Generation Capacity, Antioxidant Activity and Immunomodulatory Activities by Chinese Yang and Yin Tonifying Herbs. Chinese Medicine, 2, 3. https://doi.org/10.1186/1749-8546-2-3

[8] Wong, H.S., Leong, P.K., Chen, J., Leung, H.Y., Chan, W.M. and Ko, K.M. (2016) $\beta$-Sitosterol Increases Mitochondrial Electron Transport by Fluidizing Mitochondrial Membranes and Enhances Mitochondrial Responsiveness to Increasing Energy Demand by the Induction of Uncoupling in C2C12 Myotubes. Journal of Functional Foods, 23, 253-260. https://doi.org/10.1016/j.jff.2016.02.045

[9] Leung, H.Y. and Ko, K.M. (2008) Herba Cistanche Extract Enhances Mitochondrial ATP Generation in Rat Hearts and H9c2 Cells. Pharmaceutical Biology, 46, 418-424. https://doi.org/10.1080/13880200802055883

[10] De Paillerets, C., Gallay, J. and Alfsen, A. (1984) Effect of Cholesterol and Protein Content on Membrane Fluidity and $3 \beta$-Hydroxysteroid Dehydrogenase Activity in Mitochondrial Inner Membranes of Bovine Adrenal Cortex. B Biochimica et Biophysica Acta (BBA) - Proteins and Proteomics, 772, 183-191. https://doi.org/10.1016/0005-2736(84)90042-7

[11] Griffith, O.W. (1980) Determination of Glutathione and Glutathione Disulfide Using Glutathione Reductase and 2-Vinylpyridine. Analytical Biochemistry, 106, 207-212. https://doi.org/10.1016/0003-2697(80)90139-6

[12] Chen, J., Wong, H.S., Leong, P.K., Leung, H.Y., Chan, W.M. and Ko, K.M. (2014) New Insights into the Chemical and Biochemical Basis of the "Yang-Invigorating" Action of Chinese Yang-Tonic Herbs. Evidence-Based Complementary and Alternative Medicine, 2014, Article ID: 856273. https://doi.org/10.1155/2014/856273 
[13] Yu, H., Liu, J., Li, J., Zang, T., Luo, G. and Shen, J. (2005) Protection of Mitochondrial Integrity from Oxidative Stress by Selenium-Containing Glutathione Transferase. Applied Biochemistry and Biotechnology, 127, 133-142. https://doi.org/10.1385/ABAB:127:2:133

[14] Marí, M., Morales, A., Colell, A., García-Ruiz, C. and Fernández-Checa, J.C. (2009) Mitochondrial Glutathione, a Key Survival Antioxidant. Antioxidants \& Redox Signaling, 11, 2685-2700. https://doi.org/10.1089/ars.2009.2695

[15] Stefanson, A.L. and Bakovic, M. (2014) Dietary Regulation of Keap1/Nrf2/ARE Pathway: Focus on Plant-Derived Compounds and Trace Minerals. Nutrients, 6, 3777-3801. https://doi.org/10.3390/nu6093777

[16] Leong, P.K. and Ko, K.M. (2016) Induction of the Glutathione Antioxidant Response/Glutathione Redox Cycling by Nutraceuticals: Mechanism of Protection against Oxidant-Induced Cell Death. Current Trends in Nutraceuticals, 1, 1-9. 